\title{
Scotogenic $S_{3}$ symmetric generation of realistic neutrino mixing
}

\author{
Soumita Pramanick* \\ Harish-Chandra Research Institute, Chhatnag Road, Jhunsi, Allahabad 211019, India
}

(Received 24 April 2019; published 12 August 2019)

\begin{abstract}
Realistic neutrino mixing is achieved at a one-loop level radiatively using $S_{3} \times Z_{2}$ symmetry. The model is comprised of two right-handed neutrinos, maximally mixed to produce the structure of the left-handed Majorana neutrino mass matrix characterized by $\theta_{13}=0, \theta_{23}=\pi / 4$, and any value of $\theta_{12}^{0}$ particular to the tribimaximal (TBM), bimaximal (BM) and golden ratio (GR) or other mixings. A small deviation from this maximal mixing between the two right-handed neutrinos could generate nonzero $\theta_{13}$, shifts of the atmospheric mixing angle $\theta_{23}$ from $\pi / 4$, and also could correct the solar mixing angle $\theta_{12}$ by a small amount altogether in a single step. In this scotogenic mechanism of generating a nonzero $\theta_{13}$ by shifting from the maximal mixing in the right-handed neutrino sector, two $Z_{2}$ odd inert scalar $S U(2)_{L}$ doublets were used, the lightest of which can serve as a dark matter candidate.
\end{abstract}

DOI: 10.1103/PhysRevD.100.035009

\section{INTRODUCTION}

Neutrinos oscillate owing to their massive nature as established by the oscillation experiments. The mass eigenstates and flavor eigenstates are different and are related by the Pontecorvo, Maki, Nakagawa, SakataPMNS-matrix,

$$
U=\left(\begin{array}{ccc}
c_{12} c_{13} & s_{12} c_{13} & -s_{13} e^{-i \delta} \\
-c_{23} s_{12}+s_{23} s_{13} c_{12} e^{i \delta} & c_{23} c_{12}+s_{23} s_{13} s_{12} e^{i \delta} & -s_{23} c_{13} \\
-s_{23} s_{12}-c_{23} s_{13} c_{12} e^{i \delta} & -s_{23} c_{12}+c_{23} s_{13} s_{12} e^{i \delta} & c_{23} c_{13}
\end{array}\right)
$$

Here, $c_{i j}=\cos \theta_{i j}$ and $s_{i j}=\sin \theta_{i j}$. Needless to mention that the mass eigenstates are nondegenerate.

Nonzero $\theta_{13}$, though small in comparison to the other mixing angles, was discovered in 2012 by the shortbaseline reactor antineutrino experiments [1]. Before these nonzero $\theta_{13}$ results, models were studied in literature that correspond to the tribimaximal (TBM), bimaximal (BM), and golden ratio (GR) mixings (that we now onwards collectively refer to as popular lepton mixings). All these mixings have $\theta_{13}=0, \theta_{23}=\pi / 4$, and tuning $\theta_{12}^{0}$ to the specific values as shown in Table I produced the different mixing patterns viz. TBM, BM, and GR.

Setting $\theta_{13}=0$ and $\theta_{23}=\pi / 4$ in Eq. (1) will yield a general structure for all popular mixing as

\footnotetext{
*soumita509@gmail.com
}

Published by the American Physical Society under the terms of the Creative Commons Attribution 4.0 International license. Further distribution of this work must maintain attribution to the author(s) and the published article's title, journal citation, and DOI. Funded by SCOAP ${ }^{3}$.

$$
U^{0}=\left(\begin{array}{ccc}
\cos \theta_{12}^{0} & \sin \theta_{12}^{0} & 0 \\
-\frac{\sin \theta_{12}^{0}}{\sqrt{2}} & \frac{\cos \theta_{12}^{0}}{\sqrt{2}} & -\frac{1}{\sqrt{2}} \\
-\frac{\sin \theta_{12}^{0}}{\sqrt{2}} & \frac{\cos \theta_{12}^{0}}{\sqrt{2}} & \frac{1}{\sqrt{2}}
\end{array}\right) .
$$

The current $3 \sigma$ global fit [2,3] for $\theta_{13}, \theta_{23}$, and $\theta_{12}$ as from NuFIT3.2 of 2018 [2] are

$$
\begin{aligned}
& \theta_{12}=(31.42-36.05)^{\circ}, \\
& \theta_{23}=(40.3-51.5)^{\circ}, \\
& \theta_{13}=(8.09-8.98)^{\circ} .
\end{aligned}
$$

So popular mixing and nonzero $\theta_{13}$ observations are not in harmony. Several model-building exercises have been taking place since the observation of the nonzero $\theta_{13}$ to include it in the popular mixing framework. In [4], the possibility of the smallness of $\theta_{13}$ and $\Delta m_{\text {solar }}^{2}$ to have a common origin was explored. In some effort [5], a dominant component was characterized by larger oscillation parameters such as $\Delta m_{\mathrm{atmos}}^{2}$ and $\theta_{23}=\pi / 4$, whereas the smaller mixing parameters viz. nonzero $\theta_{13}, \theta_{12}$, solar 
TABLE I. The values $\theta_{12}^{0}$ corresponding to various popular lepton mixings namely, TBM, BM, and GR patterns.

\begin{tabular}{lccc}
\hline \hline Model & TBM & BM & GR \\
\hline$\theta_{12}^{0}$ & $35.3^{\circ}$ & $45.0^{\circ}$ & $31.7^{\circ}$ \\
\hline \hline
\end{tabular}

splitting, and deviation of atmospheric mixing from maximality were produced by a smaller seesaw [6] component as a perturbation to the dominant one. ${ }^{1}$ In $[8,9]$, the mixing angle $\theta_{13}=0$ was produced using various symmetries, and nonvanishing $\theta_{13}$ was produced by a perturbation to these symmetric forms.

The popular mixings were amended at tree level using a two-component Lagrangian with the discrete symmetries $A 4, S_{3}$ in $[10,11]$. In these models, type II seesaw yielded the dominant component that gave the popular mixing; corrections to which were offered by type I seesaw subdominant component. A similar enterprise just for the no solar mixing (NSM) case, i.e., $\theta_{12}^{0}=0$ using $A 4$ was pursued $^{2}$ in [12]. In [13], TBM was obtained radiatively using $A 4$. Recent works with realistic neutrino mixings can be found in $[14,15]$.

Here, we discuss a radiative $S_{3} \times Z_{2}$ model. $^{3}$ Some earlier works on $S_{3}$ in the context of neutrino mass are [16,17]. A neutrino mass with $S_{3} \times Z_{2}$ within left-right symmetry was studied in [18]. A common practice [19] was to find a symmetry among the three neutrinos that can produce a mass matrix that can be expressed as a linear combination of a democratic matrix $M_{\mathrm{dem}}$ and an identity matrix $I$, like $c_{1} I+c_{2} M_{\text {dem }}$ with $c_{1}$ and $c_{2}$ being two complex numbers. This could serve as a reasonable scenario to start with from which some models obtained realistic mixing through a perturbation to such initial structures [19], whereas in some models [20], various GUT symmetries or extradimensional theories were considered to generate these initial structures, and renormalization group effects at high energies were explored to obtain realistic mixing. Another way [21] of constructing $S_{3}$ models is to have a 3-3-1 local gauge symmetry and later on, associate it to a $(B-L)$ extension or use soft breaking of $S_{3}$. Since $S_{3}$ has irreducible representations of onedimension and two-dimension, the latter can be used to obtain maximal mixing in the $\nu_{\mu}-\nu_{\tau}$ block [22]. Collider signatures of $S_{3}$ flavor symmetry was vividly studied in [23]. $S_{3}$ models are also studied in the quark sector [24]. Some earlier studies on scotogenic models can be found in [25].

\footnotetext{
${ }^{1}$ For some earlier models with similar goals, see [7].

${ }^{2}$ The dominant type II seesaw had a vanishing solar splitting; thus, one can make use of degenerate perturbation theory to get a large solar mixing.

${ }^{3} \mathrm{~A}$ brief account on discrete group $S_{3}$ in presented in Appendix A of the paper.
}

In this work, our objective is to use $S_{3}$ to radiatively ${ }^{4}$ obtain

(1) The structure of the mixing matrix of a popular mixing kind as shown in Eq. (2) that is characterized by $\theta_{13}=0, \theta_{23}=\pi / 4$, and $\theta_{12}^{0}$ of any of the alternatives displayed in Table I.

(2) Realistic neutrino mixings, i.e., precisely nonzero $\theta_{13}$, shifts of the atmospheric mixing angle $\theta_{23}$ from maximality and tiny corrections to the solar mixing angle $\theta_{12}$.

In this radiative $S_{3} \times Z_{2}$ model, neutrino masses and mixings are generated at one loop. The model has two right-handed neutrinos comprising an $S_{3}$ doublet, that are maximally mixed to obtain the structure as required by popular mixings as in Eq. (2). A small deviation from this maximal mixing in the right-handed neutrino sector could produce in a single step nonzero $\theta_{13}$, shifts of $\theta_{23}$ from $\pi / 4$, and small corrections to $\theta_{12}$ as is required by the mixing to be realistic. To achieve this, two $Z_{2}$ odd scalars $\eta_{i},(i=1,2)$ were required; the lightest among them can be a good dark matter candidate. A similar analysis based on $A 4$ was performed, where instead of using deviations from maximal mixing between the two right-handed neutrino states to generate nonzero $\theta_{13}$, small mass splittings between two right-handed neutrinos were used as in [27].

\section{THE $S_{3} \times Z_{2}$ MODEL}

In the mass basis, the left-handed neutrino Majorana mass matrix is $M_{\nu L}^{\text {mass }}=\operatorname{diag}\left(m_{1}, m_{2}, m_{3}\right)$. One can transport this in its flavor basis with the help of the common form of the popular lepton mixing matrix $U^{0}$ in Eq. (2) as

$$
M_{\nu L}^{\text {flavor }}=U^{0} M_{\nu L}^{\text {mass }} U^{0 T}=\left(\begin{array}{lll}
a & c & c \\
c & b & d \\
c & d & b
\end{array}\right) .
$$

The $a, b, c$, and $d$ used here are given by

$$
\begin{aligned}
& a=m_{1} \cos ^{2} \theta_{12}^{0}+m_{2} \sin ^{2} \theta_{12}^{0} \\
& b=\frac{1}{2}\left(m_{1} \sin ^{2} \theta_{12}^{0}+m_{2} \cos ^{2} \theta_{12}^{0}+m_{3}\right) \\
& c=\frac{1}{2 \sqrt{2}} \sin 2 \theta_{12}^{0}\left(m_{2}-m_{1}\right) \\
& d=\frac{1}{2}\left(m_{1} \sin ^{2} \theta_{12}^{0}+m_{2} \cos ^{2} \theta_{12}^{0}-m_{3}\right) .
\end{aligned}
$$

Thus,

$$
\tan 2 \theta_{12}^{0}=\frac{2 \sqrt{2} c}{b+d-a}
$$

\footnotetext{
${ }^{4} \mathrm{~A}$ systematic analysis of radiative neutrino mass models can be found in [26].
} 
TABLE II. All fields along with their respective charges. We confine this model to the neutrino sector only.

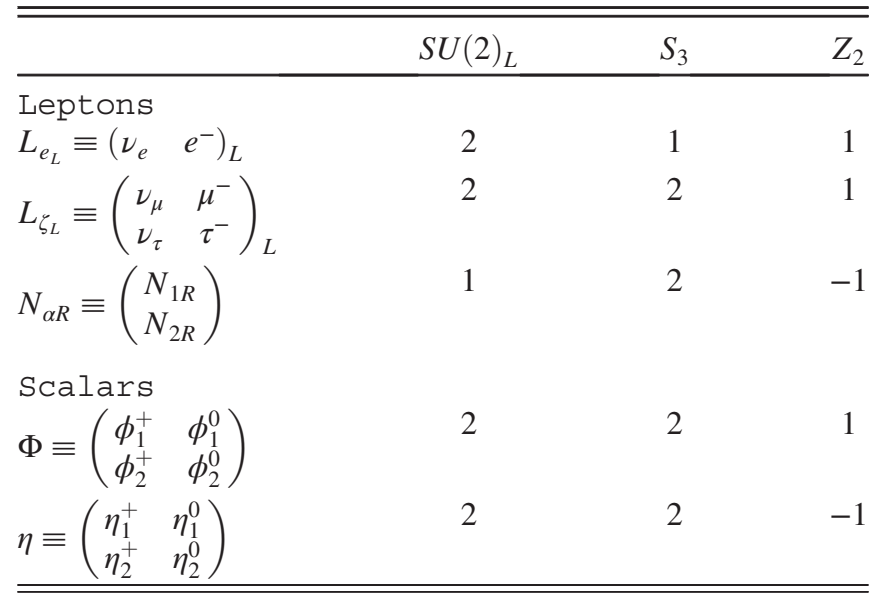

It is essential for $a, b, c$, and $d$ to be nonzero for the neutrino masses to be realistic and nondegenerate.

Our prime intent is to generate the form of $M_{\nu L}^{\text {flavor }}$ in Eq. (4) radiatively with one loop. Thus, one has to designate each of the fields in our model with particular $S_{3} \times Z_{2}$ quantum numbers. There are two right-handed neutrinos present in the model. Maximal mixing between these two right-handed neutrino fields can produce the desired form of the left-handed Majorana neutrino mass matrix in Eq. (4) that corresponds to $\theta_{13}=0, \theta_{23}=\pi / 4$, and $\theta_{12}^{0}$ of the popular lepton mixing scenarios. After obtaining the form in Eq. (4), we will see, in due course, that a slight shift from this maximal mixing between the right-handed neutrino states is capable of yielding realistic neutrino mixings, viz. a nonzero $\theta_{13}$, deviation of atmospheric mixing $\theta_{23}$ from $\pi / 4$ as well as small corrections to solar mixing $\theta_{12}$.

The model has the three left-handed lepton $S U(2)_{L}$ doublets $L_{\zeta_{L}} \equiv\left(\nu_{\zeta} \zeta^{-}\right)_{L}^{T}$, where $\zeta=e, \mu$, $\tau$, out of which $L_{\mu_{L}}$ and $L_{\tau_{L}}$ comprise a doublet of $S_{3}$ whereas $L_{e L}$ remains a singlet under $S_{3}$. Apart from these, there are two Standard Model (SM) gauge singlet right-handed neutrinos $N_{\alpha R}$, $(\alpha=1,2)$ that transform as a doublet under $S_{3}$. The scalar spectrum of the model has a couple of inert $S U(2)_{L}$ doublet scalars, $\eta_{i} \equiv\left(\eta_{i}^{+}, \eta_{i}^{0}\right)^{T},(i=1,2)$, forming an $S_{3}$ doublet $(\eta)$. We also have two other $S U(2)_{L}$ doublet scalars, namely $\Phi_{j} \equiv\left(\phi_{j}^{+}, \phi_{j}^{0}\right)^{T},(j=1,2)$, that are combined to form an $S_{3}$ doublet $(\Phi)$. Besides the $S_{3}$, the model also has an unbroken $Z_{2}$ symmetry under which all other fields except the right-handed neutrinos and the scalar $\eta$ are even. After spontaneous symmetry breaking (SSB), $\phi_{j}$ get a vacuum expectation value (vev), but $\eta_{i}$ do not. Let $v_{j}$ be the vevs of $\phi_{j}^{0}$, i.e., $\left\langle\Phi_{j}\right\rangle \equiv v_{j},(j=1,2)$. Fields and their specific charges are shown in Table II. We deal with the neutrino sector only in this model. The charged lepton mass matrix is diagonal in the basis in which we perform the analysis, and the entire mixing comes from the neutrino sector.

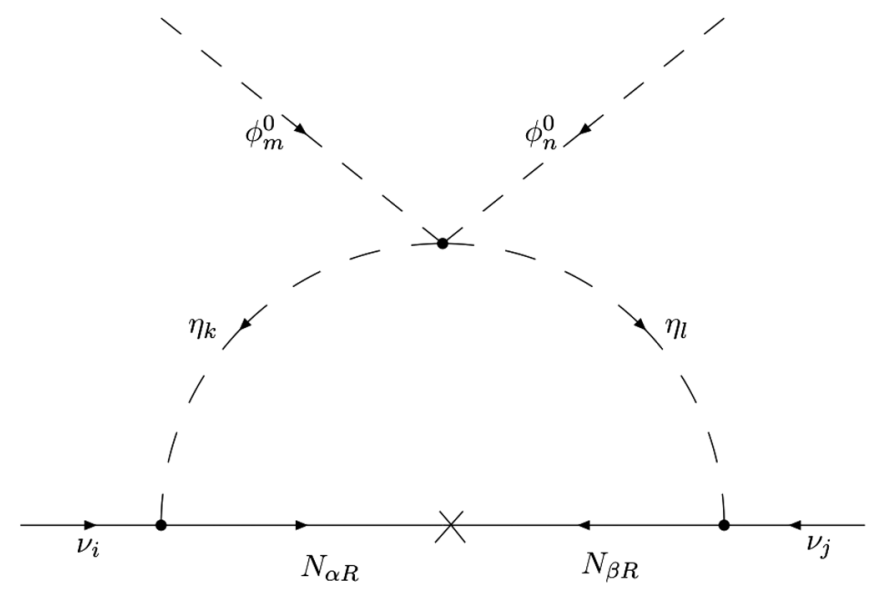

FIG. 1. One-loop scotogenic neutrino mass generation using $S_{3} \times Z_{2}$ symmetry.

Neutrino mass can be generated radiatively at the one-loop level from Fig. 1. The neutrino mass matrix will receive contributions from the following terms of the $S_{3} \times Z_{2}$ invariant scalar potential from the scalar four-point vertex ${ }^{5}$ :

$$
\begin{aligned}
V_{\text {relevant }} \supset & \lambda_{1}\left[\left\{\left(\eta_{2}^{\dagger} \phi_{2}+\eta_{1}^{\dagger} \phi_{1}\right)^{2}\right\}+\text { H.c. }\right] \\
& +\lambda_{2}\left[\left\{\left(\eta_{2}^{\dagger} \phi_{2}-\eta_{1}^{\dagger} \phi_{1}\right)^{2}\right\}+\text { H.c. }\right] \\
& +\lambda_{3}\left[\left\{\left(\eta_{1}^{\dagger} \phi_{2}\right)\left(\eta_{2}^{\dagger} \phi_{1}\right)+\left(\eta_{2}^{\dagger} \phi_{1}\right)\left(\eta_{1}^{\dagger} \phi_{2}\right)\right\}+\text { H.c. }\right] .
\end{aligned}
$$

Here, all the quartic couplings $\lambda_{j}(j=1,2,3)$ are taken real.

At all the three vertices of Fig. 1, all symmetries are conserved. The Dirac vertices conserving $S_{3} \times Z_{2}$ can be written as

$$
\begin{aligned}
\mathcal{L}_{\text {Yukawa }}= & y_{1}\left[\left(\bar{N}_{2 R} \eta_{2}^{0}+\bar{N}_{1 R} \eta_{1}^{0}\right) \nu_{e}\right] \\
& +y_{2}\left[\left(\bar{N}_{1 R} \eta_{2}^{0}\right) \nu_{\tau}+\left(\bar{N}_{2 R} \eta_{1}^{0}\right) \nu_{\mu}\right]+\text { H.c. }
\end{aligned}
$$

Since the left-handed neutrinos $\nu_{\zeta_{L}}$ transform as a doublet of $S_{3}$ for $(\zeta=\mu, \tau)$ and is invariant under $S_{3}$ if $\zeta=e$, the Yukawa couplings involved are different for $(\zeta=\mu, \tau)$ and $\zeta=e$, namely, $y_{1}$ for $\zeta=e$ and $y_{2}$ for $(\zeta=\mu, \tau)$, respectively.

Let us now have a look at the right-handed neutrino sector. Recall we have two SM gauge singlet right-handed neutrinos, $N_{1 R}$ and $N_{2 R}$, that transform as a doublet of $S_{3}$. Thus, the $S_{3} \times Z_{2}$ invariant direct mass term for the righthanded neutrinos will look like

\footnotetext{
${ }^{5}$ Two $\eta$ are created and two $\phi$ are destroyed at the scalar four point vertex causing terms of $\left(\eta^{\dagger} \phi\right)\left(\eta^{\dagger} \phi\right)$ nature to be pertinent among other terms in the scalar potential. The complete scalar potential containing all the terms can be found in Appendix B.
} 
$\mathcal{L}_{\text {right-handed neutrinos }}=\frac{1}{2} m_{R_{12}}\left[N_{1 R}^{T} C^{-1} N_{2 R}+N_{2 R}^{T} C^{-1} N_{1 R}\right]$.

Thus, the $S_{3}$ symmetry allows a symmetric mass matrix with only nonzero off diagonal terms for the right-handed neutrinos. If one allows the soft breaking of $S_{3}$ at the scale where the right-handed neutrinos get mass by introducing terms like

$$
\mathcal{L}_{\text {soft }}=\frac{1}{2}\left[m_{R_{11}} N_{1 R}^{T} C^{-1} N_{1 R}+m_{R_{22}} N_{2 R}^{T} C^{-1} N_{2 R}\right]
$$

to get nonzero diagonal entries, then one can write the right-handed neutrino mass matrix as

$$
M_{\nu_{R}}=\frac{1}{2}\left(\begin{array}{ll}
m_{R_{11}} & m_{R_{12}} \\
m_{R_{12}} & m_{R_{22}}
\end{array}\right) .
$$

The symmetric structure of the matrix in Eq. (11) also reflects its Majorana nature.

Before moving on, let us have a brief discussion about the dark matter candidates in the model. It is a common practice in literature to stabilize dark matter candidate with discrete symmetries like $Z_{2}$. Thus, the $Z_{2}$ symmetry is an indication that this model can provide dark matter candidate. Both the right-handed neutrinos and the scalar fields $\eta$ are odd under $Z_{2}$, among which $\eta$ are chosen lighter than the right-handed neutrinos $N_{\alpha R},(\alpha=1,2)$. Although from the $m_{\eta}^{2}$ term in Eq. (B1), the $\eta_{i}(i=1,2)$ appear to be degenerate in mass; since the $S_{3}$ symmetry is softly broken in the right-handed neutrino sector, it can lead to small mass splitting between the two $\eta_{i},(i=1,2)$. The lightest among the two $\eta_{i},(i=1,2)$ can be the dark matter candidate.

With the model ingredients ready, at this stage, we are in a position to present a basic description of the left-handed Majorana neutrino mass matrix arising from Fig. 1; the detailed expressions for which will be provided at a later stage of our analysis. To set the stage of the discussion, let us first sketchily indicate how the elements of the lefthanded neutrino mass matrix will receive contributions from this one-loop diagram [28] in Fig. 1. Let us make a few simplifying assumptions to make the expressions look less complicated at the moment. For this purpose, let $\lambda$ commonly represent some combinations of the three quartic couplings given in Eq. (B), i.e., $\lambda_{1}, \lambda_{2}$, and $\lambda_{3}$. Also the splitting between the masses of $\eta_{1}$ and $\eta_{2}$ comprising the $S_{3}$ doublet is neglected, and $m_{0}$ is assumed to be the common mass of them. Further, if the real part of $\eta_{j}^{0}$ is denoted by $\eta_{R j}$ and $\eta_{I j}$ be the imaginary part of $\eta_{j}^{0}$, then difference between the masses of $\eta_{R j}$ and $\eta_{I j}$ can be taken to be proportional to $\lambda v_{j}$ and can be small, in general.

It is imperative to note that under $S_{3}, \nu_{e}$ is invariant, whereas $\nu_{\zeta}(\zeta=\mu, \tau)$ transform as a doublet. This feature will manifest through the Yukawa couplings [see Eq. (8)] at the two Dirac vertices, which in its turn will dictate the structure of the left-handed neutrino mass matrix. Let $z \equiv \frac{m_{R}^{2}}{m_{0}^{2}}$, where $m_{R}$ is the average mass of the heavy right-handed neutrino states. Since $z$ always appears only in the logarithm, we do not distinguish between the masses of the different right-handed neutrinos for the purpose of defining $z$ throughout. Under this assumption, the second diagonal entry, for example, will have the form,

$$
\left(M_{\nu_{L}}^{\mathrm{flavor}}\right)_{22}=\lambda \frac{v_{m} v_{n}}{8 \pi^{2}} \frac{y_{2}^{2}}{m_{R_{22}}}[\ln z-1] .
$$

It is noteworthy that Eq. (12) is valid in the limit $m_{R}^{2} \gg m_{0}^{2}$. For $\left(M_{\nu_{L}}^{\text {flavor }}\right)_{22}$, as noted earlier in Eq. (8), $\nu_{\mu}$ couples only to $N_{2 R}$; thus, at both the Dirac vertices, $N_{2 R}$ will couple with $\nu_{\mu}$. Hence, the $(2,2)$ element of the left-handed neutrino mass matrix will get contribution from $m_{R_{22}}$ only. Also, $y_{2}$ is the only Yukawa coupling that will appear since we are dealing with $\nu_{\mu}$ at both the Dirac vertices for $\left(M_{\nu_{L}}^{\text {flavor }}\right)_{22}$. From similar arguments, one can obtain an expression for $\left(M_{\nu_{L}}^{\text {flavor }}\right)_{33}$ just by replacing $m_{R_{22}}$ by $m_{R_{11}}$ in Eq. (12).

Let us now concentrate on the off diagonal $(2,3)$ entry. Thus, one has to consider $\nu_{\mu}$ at one of the Dirac vertices and $\nu_{\tau}$ at the other. From Eq. (8), one can note that $\nu_{\mu}$ couples to $N_{2 R}$ only, whereas $\nu_{\tau}$ does so with $N_{1 R}$. Thus, at one of the Dirac vertices, we will have $N_{1 R}$ and $N_{2 R}$ at the other. Therefore, off diagonal entries from right-handed neutrino mass matrix will come into play, and $\left(M_{\nu_{L}}^{\text {flavor }}\right)_{23}$ will get contributions from $m_{R_{12}}$ in addition to that from $m_{R_{11}}$ and $m_{R_{22}}$. Needless to mention that the Yukawa coupling involved will be $y_{2}$ as can be seen from Eq. (8). Thus, one can write

$$
\left(M_{\nu_{L}}^{\text {flavor }}\right)_{23}=\lambda \frac{v_{m} v_{n}}{8 \pi^{2}} \frac{y_{2}^{2} m_{R_{12}}}{m_{R_{11}} m_{R_{22}}}[\ln z-1] .
$$

While writing down Eq. (13), we are taking into account the mass insertion approximation. In a similar spirit, one can write down expressions for $(1,1),(1,2)$, and the $(1,3)$ entries of the left-handed Majorana neutrino mass matrix.

For notational ease, let us absorb everything else present in the rhs of expressions for the elements of the left-handed Majorana neutrino mass matrix, as in Eq. (12) and Eq. (13) except for the Yukawa couplings, quartic couplings, and the vevs in loop contributing factors say $r_{\alpha \beta}$ given by

$$
\begin{aligned}
& r_{11} \equiv \frac{1}{8 \pi^{2} m_{R_{11}}}[\ln z-1], \\
& r_{22} \equiv \frac{1}{8 \pi^{2} m_{R_{22}}}[\ln z-1], \\
& r_{12} \equiv \frac{m_{R_{12}}}{8 \pi^{2} m_{R_{11}} m_{R_{22}}}[\ln z-1] .
\end{aligned}
$$


From Eqs. (12), (13), (14), and (7), the left-handed neutrino Majorana mass matrix radiatively generated at one loop as shown in Fig. 1 is

$$
M_{\nu_{L}}^{\text {flavor }}=\left(\begin{array}{ccc}
\chi_{1} & \chi_{4} & \chi_{5} \\
\chi_{4} & \chi_{2} & \chi_{6} \\
\chi_{5} & \chi_{6} & \chi_{3}
\end{array}\right)
$$

where

$$
\begin{aligned}
& \chi_{1} \equiv y_{1}^{2}\left[4 r_{12} v_{1} v_{2}\left(\lambda_{3}+\lambda_{1}-\lambda_{2}\right)+\left(r_{11} v_{1}^{2}+r_{22} v_{2}^{2}\right)\left(\lambda_{1}+\lambda_{2}\right)\right] \\
& \chi_{2} \equiv y_{2}^{2}\left[r_{22}\left(\lambda_{1}+\lambda_{2}\right) v_{1}^{2}\right] \\
& \chi_{3} \equiv y_{2}^{2}\left[r_{11}\left(\lambda_{1}+\lambda_{2}\right) v_{2}^{2}\right] \\
& \chi_{4} \equiv y_{1} y_{2}\left[r_{12}\left(\lambda_{1}+\lambda_{2}\right) v_{1}^{2}+2 r_{22}\left(\lambda_{3}+\lambda_{1}-\lambda_{2}\right) v_{1} v_{2}\right] \\
& \chi_{5} \equiv y_{1} y_{2}\left[r_{12}\left(\lambda_{1}+\lambda_{2}\right) v_{2}^{2}+2 r_{11}\left(\lambda_{3}+\lambda_{1}-\lambda_{2}\right) v_{1} v_{2}\right] \\
& \chi_{6} \equiv y_{2}^{2}\left[2 r_{12}\left(\lambda_{3}+\lambda_{1}-\lambda_{2}\right) v_{1} v_{2}\right]
\end{aligned}
$$

Here, $\left\langle\Phi_{j}\right\rangle \equiv v_{j}$ with $(j=1,2)$.
For the left-handed neutrino mass matrix in Eq. (15) to be of the form of Eq. (4), i.e., the structure needed for $\theta_{13}=0, \theta_{23}=\pi / 4$, and $\theta_{12}^{0}$ of the popular mixing kind, we have to set $\chi_{1} \neq \chi_{2}=\chi_{3}$ as well as $\chi_{4}=\chi_{5}$. This is achieved when $v_{1}=v_{2}=v$ and $r_{11}=r_{22}=r$. The condition $r_{11}=r_{22}=r$ when translated in terms of the righthanded neutrino mass matrix in Eq. (11) using Eq. (14) will lead to

$$
M_{\nu_{R}}=\frac{1}{2}\left(\begin{array}{ll}
m_{R_{11}} & m_{R_{12}} \\
m_{R_{12}} & m_{R_{11}}
\end{array}\right) .
$$

The matrix in Eq. (17) corresponds to maximal mixing in the right-handed neutrino sector. Thus, to get the form of left-handed neutrino mass matrix as in Eq. (4), it is necessary to have $v_{1}=v_{2}=v$ as well as maximal mixing between $N_{1 R}$ and $N_{2 R}$; i.e., we have to set $r_{11}=r_{22}=r$. Implementing these constraints to the general form of the mass matrix in Eq. (15), we get

$$
M_{\nu_{L}}^{\text {flavor }}=v^{2}\left(\begin{array}{ccc}
y_{1}^{2}\left[4 r_{12} \lambda_{123}+2 r \lambda_{12}\right] & y_{1} y_{2}\left[r_{12} \lambda_{12}+2 r \lambda_{123}\right] & y_{1} y_{2}\left[r_{12} \lambda_{12}+2 r \lambda_{123}\right] \\
y_{1} y_{2}\left[r_{12} \lambda_{12}+2 r \lambda_{123}\right] & y_{2}^{2} r \lambda_{12} & y_{2}^{2}\left(2 r_{12} \lambda_{123}\right) \\
y_{1} y_{2}\left[r_{12} \lambda_{12}+2 r \lambda_{123}\right] & y_{2}^{2}\left(2 r_{12} \lambda_{123}\right) & y_{2}^{2} r \lambda_{12}
\end{array}\right) \text {. }
$$

Here, $\lambda_{12} \equiv \lambda_{1}+\lambda_{2}$ and $\lambda_{123} \equiv \lambda_{3}+\lambda_{1}-\lambda_{2}$. To get the form of $M_{\nu_{L}}^{\text {flavor }}$ in Eq. (4), one has to identify

$$
\begin{aligned}
a & \equiv y_{1}^{2} v^{2}\left[4 r_{12} \lambda_{123}+2 r \lambda_{12}\right] \\
& =y_{1}^{2} v^{2}\left[4 r_{12}\left(\lambda_{3}+\lambda_{1}-\lambda_{2}\right)+2 r\left(\lambda_{1}+\lambda_{2}\right)\right] \\
b & \equiv y_{2}^{2} v^{2} r \lambda_{12}=y_{2}^{2} v^{2} r\left(\lambda_{1}+\lambda_{2}\right) \\
c & \equiv y_{1} y_{2} v^{2}\left[r_{12} \lambda_{12}+2 r \lambda_{123}\right] \\
& =y_{1} y_{2} v^{2}\left[r_{12}\left(\lambda_{1}+\lambda_{2}\right)+2 r\left(\lambda_{3}+\lambda_{1}-\lambda_{2}\right)\right] \\
d & \equiv y_{2}^{2} v^{2}\left(2 r_{12} \lambda_{123}\right)=y_{2}^{2} v^{2}\left[2 r_{12}\left(\lambda_{3}+\lambda_{1}-\lambda_{2}\right)\right] .
\end{aligned}
$$

So far, we are able to obtain the form of left-handed neutrino mass matrix required for $\theta_{13}=0, \theta_{23}=\pi / 4$, and $\theta_{12}^{0}$ of the popular mixing varieties. With this in hand, the obvious follow-up enterprise, as mentioned earlier, will be to obtain realistic mixing viz. nonzero $\theta_{13}$, deviations of the atmospheric mixing angle $\theta_{23}$ from $\pi / 4$ as well as tiny corrections to $\theta_{12}$ also. To get such realistic neutrino mixing, we have to shift from the choice of $r_{11}=r_{22}=r$, i.e., allow the two diagonal entries of the right-handed neutrino mass matrix to slightly differ from each other. In other words, let $r_{22}=r_{11}+\epsilon$, where $\epsilon$ is a small quantity. Therefore, one gets back the general form of $M_{\nu R}$ in Eq. (11) characterized by nonmaximal mixing between $N_{1 R}$ and $N_{2 R}$. Thus, setting $r_{22}=r_{11}+\epsilon$ is precisely shifting from the maximal mixing between the two right-handed neutrino states. With $v_{1}=$ $v_{2}=v$ still valid, we can get a dominant component of $M_{\nu_{L}}^{\text {flavor }}$ as in Eq. (18) denoted $M^{0}$ and a smaller contribution $M^{\prime}$ proportional to $\epsilon$. Hence,

$$
M_{\nu_{L}}^{\text {flavor }}=M^{0}+M^{\prime}
$$

with

$$
M^{0}=v^{2}\left(\begin{array}{ccc}
y_{1}^{2}\left[4 r_{12} \lambda_{123}+2 r_{11} \lambda_{12}\right] & y_{1} y_{2}\left[r_{12} \lambda_{12}+2 r_{11} \lambda_{123}\right] & y_{1} y_{2}\left[r_{12} \lambda_{12}+2 r_{11} \lambda_{123}\right] \\
y_{1} y_{2}\left[r_{12} \lambda_{12}+2 r_{11} \lambda_{123}\right] & y_{2}^{2} r_{11} \lambda_{12} & y_{2}^{2}\left(2 r_{12} \lambda_{123}\right) \\
y_{1} y_{2}\left[r_{12} \lambda_{12}+2 r_{11} \lambda_{123}\right] & y_{2}^{2}\left(2 r_{12} \lambda_{123}\right) & y_{2}^{2} r_{11} \lambda_{12}
\end{array}\right) \text {, }
$$


and

$$
M^{\prime}=\epsilon\left(\begin{array}{ccc}
x & y & 0 \\
y & x^{\prime} & 0 \\
0 & 0 & 0
\end{array}\right)
$$

where

$$
\begin{aligned}
x & \equiv y_{1}^{2} v^{2} \lambda_{12}=y_{1}^{2} v^{2}\left(\lambda_{1}+\lambda_{2}\right) \\
x^{\prime} & \equiv y_{2}^{2} v^{2} \lambda_{12}=y_{2}^{2} v^{2}\left(\lambda_{1}+\lambda_{2}\right) \\
y & \equiv y_{1} y_{2} v^{2} \lambda_{123}=y_{1} y_{2} v^{2}\left(\lambda_{3}+\lambda_{1}-\lambda_{2}\right) .
\end{aligned}
$$

$M^{0}$ in Eq. (21) will represent the form of left-handed neutrino mass matrix needed for $\theta_{13}=0, \theta_{23}=\pi / 4$, and $\theta_{12}^{0}$ of the popular mixing types as in Eq. (4) when we identify

$$
\begin{aligned}
a^{\prime} & \equiv y_{1}^{2} v^{2}\left[4 r_{12} \lambda_{123}+2 r_{11} \lambda_{12}\right] \\
& =y_{1}^{2} v^{2}\left[4 r_{12}\left(\lambda_{3}+\lambda_{1}-\lambda_{2}\right)+2 r_{11}\left(\lambda_{1}+\lambda_{2}\right)\right] \\
b^{\prime} & \equiv y_{2}^{2} v^{2} r_{11} \lambda_{12}=y_{2}^{2} v^{2} r_{11}\left(\lambda_{1}+\lambda_{2}\right) \\
c^{\prime} & \equiv y_{1} y_{2} v^{2}\left[r_{12} \lambda_{12}+2 r_{11} \lambda_{123}\right] \\
& =y_{1} y_{2} v^{2}\left[r_{12}\left(\lambda_{1}+\lambda_{2}\right)+2 r_{11}\left(\lambda_{3}+\lambda_{1}-\lambda_{2}\right)\right] \\
d^{\prime} & \equiv y_{2}^{2} v^{2}\left(2 r_{12} \lambda_{123}\right)=y_{2}^{2} v^{2}\left[2 r_{12}\left(\lambda_{3}+\lambda_{1}-\lambda_{2}\right)\right]
\end{aligned}
$$

in the same spirit ${ }^{6}$ as was done in case of Eq. (19).

With the help of a nondegenerate perturbation theory, we can calculate the corrections to eigenvalues and eigenvectors of $M^{0}$ from $M^{\prime}$. The unperturbed flavor basis is given by the columns of the mixing matrix $U^{0}$ as shown in Eq. (2). For ease of presentation, it is useful to define

$\gamma \equiv\left(b^{\prime}-3 d^{\prime}-a^{\prime}\right) \quad$ and

$\rho \equiv \sqrt{a^{\prime 2}+b^{\prime 2}+8 c^{\prime 2}+d^{\prime 2}-2 a^{\prime} b^{\prime}-2 a^{\prime} d^{\prime}+2 b^{\prime} d^{\prime}}$.

Thus, the third ket after receiving first order corrections will take the form,

$$
\left|\psi_{3}\right\rangle=\left(\begin{array}{c}
\frac{\epsilon}{\gamma^{2}-\rho^{2}}\left[\rho\left(\sqrt{2} y \cos 2 \theta_{12}^{0}-x^{\prime} \sin 2 \theta_{12}^{0}\right)-\gamma \sqrt{2} y\right] \\
-\frac{1}{\sqrt{2}}[1+\xi \epsilon] \\
\frac{1}{\sqrt{2}}[1-\xi \epsilon]
\end{array}\right) .
$$

Here, we have used

\footnotetext{
${ }^{6} \mathrm{We}$ are introducing the primed notation to differentiate from the $r_{11}=r_{22}=r$ case.
}

$\xi \equiv\left[\gamma x^{\prime}+\rho\left(x^{\prime} \cos 2 \theta_{12}^{0}+\sqrt{2} y \sin 2 \theta_{12}^{0}\right)\right] /\left(\gamma^{2}-\rho^{2}\right)$.

If we consider a $C P$-conserving scenario then,

$\sin \theta_{13}=\frac{\epsilon}{\gamma^{2}-\rho^{2}}\left[\rho\left(\sqrt{2} y \cos 2 \theta_{12}^{0}-x^{\prime} \sin 2 \theta_{12}^{0}\right)-\gamma \sqrt{2} y\right]$.

The expression for a nonzero $\theta_{13}$ in terms of the parameters of our model viz. $\epsilon$, the vacuum expectation values $v$ and the quartic couplings $\lambda_{i},(i=1,2,3)$, can be obtained with help of Eqs. (24), (25), and (28).

The shift of $\theta_{23}$ from $\pi / 4$ can be found from Eq. (26) as

$$
\tan \varphi \equiv \tan \left(\theta_{23}-\pi / 4\right)=\xi \epsilon .
$$

The first-order corrections to the first and second ket will contribute to changes in $\theta_{12}$. Defining

$$
\beta \equiv \frac{\left[\frac{y}{\sqrt{2}} \cos 2 \theta_{12}^{0}+\frac{1}{2}\left(x-\frac{x^{\prime}}{2}\right) \sin 2 \theta_{12}^{0}\right]}{\rho}
$$

will lead to corrected solar mixing angle given by,

$$
\tan \theta_{12}=\frac{\sin \theta_{12}^{0}+\epsilon \beta \cos \theta_{12}^{0}}{\cos \theta_{12}^{0}-\epsilon \beta \sin \theta_{12}^{0}} .
$$

Needless to mention, expressions for the corrected $\theta_{12}$ in Eq. (31) and deviations of $\theta_{23}$ from maximal mixing in Eq. (29) can be translated in terms of parameters of this $S_{3} \times Z_{2}$ symmetric model by applying Eqs. (24), (25), (27), and (30).

In our entire analysis, we have taken $r_{\alpha \beta},(\alpha, \beta=1,2)$, to be real therefore allowing no $C P$ violation. But one can associate Majorana phases to masses of the right-handed neutrinos; thus, $r_{\alpha \beta}$ can be complex quantities. Therefore, $\epsilon$ can also be complex that can give rise to $C P$ violation from Eq. (26).

Finally, we want to make a remark on the flavor changing decays of the charged leptons. For a charged lepton flavor violation (LFV), one requires the part of the Yukawa Lagrangian similar to Eq. (8),

$$
\begin{aligned}
\mathcal{L}_{\mathrm{LFV}}= & y_{1}\left[\left(\bar{N}_{2 R} \eta_{2}^{+}+\bar{N}_{1 R} \eta_{1}^{+}\right) e^{-}\right] \\
& +y_{2}\left[\left(\bar{N}_{1 R} \eta_{2}^{+}\right) \tau^{-}+\left(\bar{N}_{2 R} \eta_{1}^{+}\right) \mu^{-}\right]+\text {H.c. }
\end{aligned}
$$

At a one-loop level, LFV processes can take place through diagrams as shown in Fig. 2. From Eq. (32), it is readily seen that the $\mu^{-} \rightarrow e^{-} \gamma, \tau^{-} \rightarrow e^{-} \gamma$, and $\tau^{-} \rightarrow \mu^{-} \gamma$ processes in Fig. 2 are disallowed in the model. Specifically, the $\eta_{i}$ and $N_{\alpha}$ fields needed at the two Yukawa vertices in Fig. 2 for these LFV processes to occur can never be matched, taking into account Eq. (32). Thus, these LFV processes are 


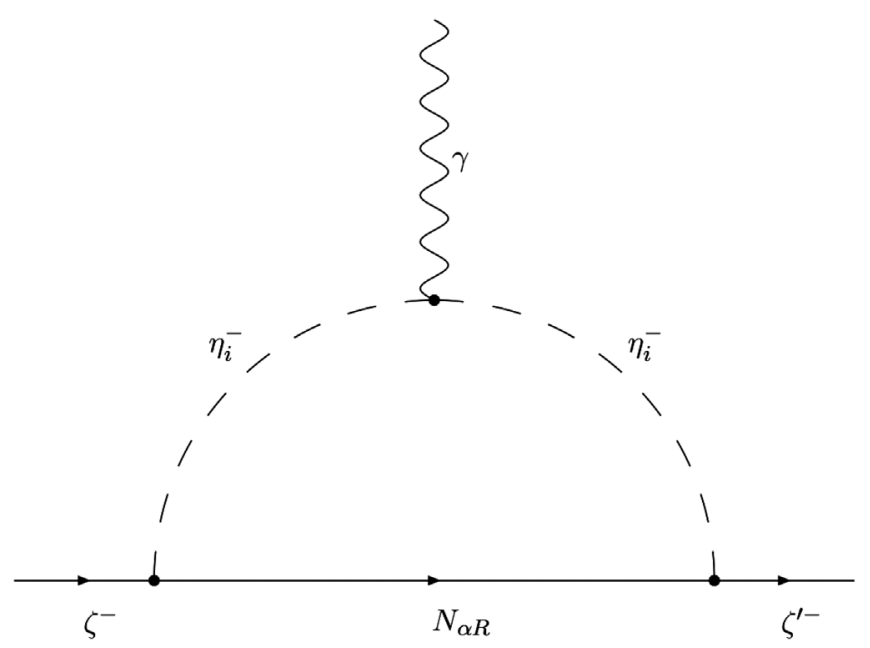

FIG. 2. Decays of the charged leptons at one loop. Here, $\zeta^{-}$and $\zeta^{\prime-}$ stands for $\left(e^{-}, \mu^{-}, \tau^{-}\right)$. For charged lepton flavor violating (LFV) processes, $\zeta^{-} \neq \zeta^{\prime-}$. Kinematically, only $\mu^{-} \rightarrow e^{-} \gamma, \tau^{-} \rightarrow$ $e^{-} \gamma$ and $\tau^{-} \rightarrow \mu^{-} \gamma$ are allowed and are therefore searched for. $S_{3}$ symmetry forbids LFV processes at a one-loop level in this model.

identically zero at the one-loop level as long as the $S_{3}$ symmetry is conserved ${ }^{7}$.

\section{CONCLUSION}

In a nutshell, a radiative $S_{3} \times Z_{2}$ symmetric scheme of scotogenic generation of realistic neutrino mixing is put forward. The model has two right-handed neutrinos, $N_{1 R}$ and $N_{2 R}$, which when maximally mixed can radiatively yield the form of a left-handed Majorana neutrino mass matrix at one loop characterized by $\theta_{13}=0, \theta_{23}=\pi / 4$, and $\theta_{12}^{0}$ of any of the values specific to the tribimaximal (TBM), bimaximal (BM), and golden ratio (GR) mixing, collectively termed as popular lepton mixings. A small deviation from maximal mixing between the two right-handed neutrino states can produce realistic mixing angles, i.e., nonzero $\theta_{13}$, shifts of the atmospheric mixing angle $\theta_{23}$ from $\pi / 4$ and small corrections to $\theta_{12}$. There are two inert $S U(2)_{L}$ doublet scalar fields $\eta_{i},(i=1,2)$ in the model. Since the $\eta_{i}$ are odd under the action of the unbroken $Z_{2}$, the lightest among these two scalars can serve as dark matter.

\section{ACKNOWLEDGMENTS}

My sincere thanks to Professor Amitava Raychaudhuri for discussions and valuable suggestions.

\section{APPENDIX A: THE GROUP $S_{3}$}

It is the permutation group of three objects [29] and therefore, has $3 !=6$ elements. $S_{3}$ has two generators $A$ and

\footnotetext{
${ }^{7}$ Since neutrino masses are small, one can neglect the LFV processes mediated by the $W$ boson.
}

TABLE III. The group table of the discrete symmetry $S_{3}$.

\begin{tabular}{lcccccc}
\hline \hline & $I$ & $A$ & $B$ & $C$ & $D$ & $F$ \\
\hline$I$ & $I$ & $A$ & $B$ & $C$ & $D$ & $F$ \\
$A$ & $A$ & $I$ & $C$ & $B$ & $F$ & $D$ \\
$F$ & $F$ & $C$ & $I$ & $D$ & $A$ & $B$ \\
$C$ & $C$ & $F$ & $D$ & $I$ & $B$ & $A$ \\
$D$ & $D$ & $B$ & $A$ & $F$ & $I$ & $C$ \\
$B$ & $B$ & $D$ & $F$ & $A$ & $C$ & $I$ \\
\hline \hline
\end{tabular}

$B$ that satisfy $A^{2}=I=B^{3}$ and $(A B)(A B)=I$. The group properties can be clearly understood from the group table shown in Table III.

It has two one-dimensional representations 1 and $1^{\prime}$, as well as one two-dimensional representation 2 . The onedimensional representation 1 is immune to both $A$ and $B$, whereas $1^{\prime}$ flips sign when acted by $A$. In two-dimensions, the group can be represented by the following matrices that obey all the properties discussed so far:

$I=\left(\begin{array}{ll}1 & 0 \\ 0 & 1\end{array}\right), \quad A=\left(\begin{array}{ll}0 & 1 \\ 1 & 0\end{array}\right), \quad B=\left(\begin{array}{cc}\omega & 0 \\ 0 & \omega^{2}\end{array}\right)$.

Here, $\omega=e^{2 \pi i / 3}$ is a cube root of one. With the generators in Eq. (A1), we can construct the rest of the members of the group as

$$
\begin{aligned}
C & =\left(\begin{array}{cc}
0 & \omega^{2} \\
\omega & 0
\end{array}\right), \\
F & =\left(\begin{array}{cc}
\omega^{2} & 0 \\
0 & \omega
\end{array}\right) .
\end{aligned}
$$

$S_{3}$ is characterized by the following product rules:

$1 \times 1^{\prime}=1^{\prime}, \quad 1^{\prime} \times 1^{\prime}=1, \quad$ and $\quad 2 \times 2=2+1+1^{\prime}$.

All the matrices $M_{i j}$ in Eqs. (A1) and (A2) obey

$$
\sum_{j, l=1,2} \alpha_{j l} M_{i j} M_{k l}=\alpha_{i k}
$$

Here, $\alpha_{i j}=0$ if $i=j$ and $\alpha_{i j}=1$ if $i \neq j$.

Let $\Phi \equiv\left(\begin{array}{l}\phi_{1} \\ \phi_{2}\end{array}\right)$ and $\Psi \equiv\left(\begin{array}{l}\psi_{1} \\ \psi_{2}\end{array}\right)$ be two doublets of $S_{3}$ which when combined according to Eq. (A3) will yield

$$
\begin{aligned}
\phi_{1} \psi_{2}+\phi_{2} \psi_{1} & \equiv 1, \quad \phi_{1} \psi_{2}-\phi_{2} \psi_{1} \equiv 1^{\prime}, \quad \text { and } \\
\left(\begin{array}{l}
\phi_{2} \psi_{2} \\
\phi_{1} \psi_{1}
\end{array}\right) & \equiv 2 .
\end{aligned}
$$

Often, we have to work with Hermitian conjugate of the fields. Owing to the properties of the complex 
representations of $S_{3}$, [say, as for $B$ displayed in Eq. (A1)], the Hermitian conjugate of $\Phi$ is given by $\Phi^{\dagger} \equiv\left(\begin{array}{c}\phi_{2}^{\dagger} \\ \phi_{1}^{\dagger}\end{array}\right)$. This $\Phi^{\dagger}$ when combined with $\Psi$, keeping Eq. (A3) in mind, we get

$$
\begin{aligned}
\phi_{2}^{\dagger} \psi_{2}+\phi_{1}^{\dagger} \psi_{1} & \equiv 1, \quad \phi_{2}^{\dagger} \psi_{2}-\phi_{1}^{\dagger} \psi_{1} \equiv 1^{\prime} \text { and } \\
\left(\begin{array}{c}
\phi_{1}^{\dagger} \psi_{2} \\
\phi_{2}^{\dagger} \psi_{1}
\end{array}\right) & \equiv 2 .
\end{aligned}
$$

Equations (A5) and (A6) play a pivotal role in determining the structure of the mass matrices in the model.
APPENDIX B: THE SCALAR POTENTIAL

The scalar sector of the model, as can be seen from Table II, is comprised of two inert $S U(2)_{L}$ doublets, $\eta_{i} \equiv\left(\eta_{i}^{+} \eta_{i}^{0}\right)^{T}, \quad(i=1,2)$, forming a doublet under $S_{3}$ denoted by $\eta$ and two other $S U(2)_{L}$ doublet scalar fields $\Phi_{j} \equiv\left(\phi_{j}^{+} \phi_{j}^{0}\right)^{T},(j=1,2)$, represented by $\Phi$, transforming as a doublet under $S_{3}$. Under the unbroken $Z_{2}, \eta$ is odd, whereas $\Phi$ is even. Thus, after $\mathrm{SSB}, \phi_{j}^{0}$ can acquire vevs $v_{j}$, $(j=1,2)$, but the $\eta_{i}^{0}$ cannot. The complete scalar potential consisting of all the terms allowed by the SM gauge symmetry and $S_{3} \times Z_{2}$ is given by

$$
\begin{aligned}
V_{\text {total }}= & m_{\eta}^{2}\left(\eta_{2}^{\dagger} \eta_{2}+\eta_{1}^{\dagger} \eta_{1}\right)+m_{\phi}^{2}\left(\phi_{2}^{\dagger} \phi_{2}+\phi_{1}^{\dagger} \phi_{1}\right)+\tilde{\lambda}_{1}\left(\eta_{2}^{\dagger} \eta_{2}+\eta_{1}^{\dagger} \eta_{1}\right)^{2}+\tilde{\lambda}_{2}\left(\eta_{2}^{\dagger} \eta_{2}-\eta_{1}^{\dagger} \eta_{1}\right)^{2}+\tilde{\lambda}_{3}\left(\phi_{2}^{\dagger} \phi_{2}+\phi_{1}^{\dagger} \phi_{1}\right)^{2} \\
& +\tilde{\lambda}_{4}\left(\phi_{2}^{\dagger} \phi_{2}-\phi_{1}^{\dagger} \phi_{1}\right)^{2}+\tilde{\lambda}_{5}\left[\left(\eta_{2}^{\dagger} \eta_{2}+\eta_{1}^{\dagger} \eta_{1}\right)\left(\phi_{2}^{\dagger} \phi_{2}+\phi_{1}^{\dagger} \phi_{1}\right)\right]+\tilde{\lambda}_{6}\left[\left(\eta_{2}^{\dagger} \eta_{2}-\eta_{1}^{\dagger} \eta_{1}\right)\left(\phi_{2}^{\dagger} \phi_{2}-\phi_{1}^{\dagger} \phi_{1}\right)\right] \\
& +\tilde{\lambda}_{7}\left[\left(\phi_{1}^{\dagger} \phi_{2}\right)\left(\phi_{2}^{\dagger} \phi_{1}\right)\right]+\tilde{\lambda}_{8}\left[\left(\eta_{1}^{\dagger} \eta_{2}\right)\left(\eta_{2}^{\dagger} \eta_{1}\right)\right]+\tilde{\lambda}_{9}\left[\left\{\left(\phi_{1}^{\dagger} \phi_{2}\right)\left(\eta_{2}^{\dagger} \eta_{1}\right)\right\}+\left\{\left(\phi_{2}^{\dagger} \phi_{1}\right)\left(\eta_{1}^{\dagger} \eta_{2}\right)\right\}\right]+V_{\text {relevant }},
\end{aligned}
$$

where

$$
\begin{aligned}
V_{\text {relevant }}= & \lambda_{1}\left[\left\{\left(\eta_{2}^{\dagger} \phi_{2}+\eta_{1}^{\dagger} \phi_{1}\right)^{2}\right\}+\text { H.c. }\right] \\
& +\lambda_{2}\left[\left\{\left(\eta_{2}^{\dagger} \phi_{2}-\eta_{1}^{\dagger} \phi_{1}\right)^{2}\right\}+\text { H.c. }\right] \\
& +\lambda_{3}\left[\left\{\left(\eta_{1}^{\dagger} \phi_{2}\right)\left(\eta_{2}^{\dagger} \phi_{1}\right)+\left(\eta_{2}^{\dagger} \phi_{1}\right)\left(\eta_{1}^{\dagger} \phi_{2}\right)\right\}+\text { H.c. }\right] .
\end{aligned}
$$

Since at the four-point scalar vertex in Fig. 1, two $\phi$ are destroyed and two $\eta$ are created, the terms only of $\left(\eta^{\dagger} \phi\right)\left(\eta^{\dagger} \phi\right)$ type play a crucial role in determining the neutrino mass matrix. Thus, we call these terms as the relevant part of the scalar potential, represented by $V_{\text {relevant }}$ in Eq. (B2). The quartic couplings $\lambda_{j}(j=1,2,3)$ appearing in Eq. (B2) were taken to be real for the analysis.
[1] For the present status of $\theta_{13}$ see presentations from Double Chooz, RENO, Daya Bay, and T2K at Neutrino 2016, http:// neutrino2016.iopconfs.org/programme.

[2] M. C. Gonzalez-Garcia, M. Maltoni, J. Salvado, and T. Schwetz, J. High Energy Phys. 12 (2012) 123.

[3] D. V. Forero, M. Tortola, and J. W. F. Valle, Phys. Rev. D 86, 073012 (2012).

[4] B. Brahmachari and A. Raychaudhuri, Phys. Rev. D 86, 051302 (2012); S. Pramanick and A. Raychaudhuri, Phys. Rev. D 88, 093009 (2013).

[5] S. Pramanick and A. Raychaudhuri, Phys. Lett. B 746, 237 (2015); Int. J. Mod. Phys. A 30, 1530036 (2015).

[6] P. Minkowski, Phys. Lett. B 67, 421 (1977); M. Gell-Mann, P. Ramond, and R. Slansky, in Supergravity, edited by F. van Nieuwenhuizen and D. Freedman (North Holland, Amsterdam, 1979), p. 315; T. Yanagida, Proc. of the Workshop on Unified Theory and the Baryon Number of the Universe (KEK, Japan, 1979); S. L. Glashow, NATO Sci. Ser. B 59, 687 (1980); R. N. Mohapatra and G. Senjanović, Phys. Rev. D 23, 165 (1981); J. Schechter and J. W. F. Valle, Phys. Rev. D 25, 774 (1982); J. Schechter and J. W. F. Valle, Phys. Rev. D 22, 2227 (1980).
[7] F. Vissani, J. High Energy Phys. 11 (1998) 025; Models with somewhat similar points of view as those espoused here are E. K. Akhmedov, Phys. Lett. B 467, 95 (1999); M. Lindner and W. Rodejohann, J. High Energy Phys. 05 (2007) 089.

[8] For other recent work after the determination of $\theta_{13}$ see $\mathrm{S}$. Antusch, S. F. King, C. Luhn, and M. Spinrath, Nucl. Phys. B856, 328 (2012); B. Adhikary, A. Ghosal, and P. Roy, Int. J. Mod. Phys. A 28, 1350118 (2013); D. Aristizabal Sierra, I. de Medeiros Varzielas, and E. Houet, Phys. Rev. D 87, 093009 (2013); R. Dutta, U. Ch, A. K. Giri, and N. Sahu, Int. J. Mod. Phys. A 29, 1450113 (2014); L. J. Hall and G. G. Ross, J. High Energy Phys. 11 (2013) 091; T. Araki, Prog. Theor. Exp. Phys. 2013, 103 B02 (2013); A. E. Carcamo Hernandez, I. de Medeiros Varzielas, S. G. Kovalenko, H. Päs, and I. Schmidt, Phys. Rev. D 88, 076014 (2013); M.-C. Chen, J. Huang, K. T. Mahanthappa, and A. M. Wijangco, J. High Energy Phys. 10 (2013) 112; B. Brahmachari and P. Roy, J. High Energy Phys. 02 (2015) 135; P. S. Bhupal Dev, B. Dutta, R. N. Mohapatra, and M. Severson, Phys. Rev. D 86, 035002 (2012). 
[9] For a review see, for example, S. F. King and C. Luhn, Rep. Prog. Phys. 76, 056201 (2013).

[10] S. Pramanick and A. Raychaudhuri, Phys. Rev. D 94, 115028 (2016).

[11] S. Pramanick, Phys. Rev. D 98, 075016 (2018).

[12] S. Pramanick and A. Raychaudhuri, Phys. Rev. D 93, 033007 (2016).

[13] E. Ma, Phys. Lett. B 671, 366 (2009).

[14] E. Ma and D. Wegman, Phys. Rev. Lett. 107, 061803 (2011); S. Gupta, A. S. Joshipura, and K. M. Patel, Phys. Rev. D 85, 031903 (2012); G. C. Branco, R. G. Felipe, F. R. Joaquim, and H. Serodio, Phys. Rev. D 86, 076008 (2012); B. Adhikary, B. Brahmachari, A. Ghosal, E. Ma, and M. K. Parida, Phys. Lett. B 638, 345 (2006); B. Karmakar and A. Sil, Phys. Rev. D 91, 013004 (2015); E. Ma, Phys. Lett. B 752, 198 (2016); X. G. He, Y. Y. Keum, and R. R. Volkas, J. High Energy Phys. 04 (2006) 039.

[15] S. K. Kang and M. Tanimoto, Phys. Rev. D 91, 073010 (2015).

[16] See, for example, P. F. Harrison and W. G. Scott, Phys. Lett. B 557, 76 (2003).

[17] S. L. Chen, M. Frigerio, and E. Ma, Phys. Rev. D 70, 073008 (2004); 70, 079905(E) (2004); E. Ma, Phys. Rev. D 61, 033012 (2000); 44, R587 (1991).

[18] J. C. Gómez-Izquierdo, Eur. Phys. J. C 77, 551 (2017); E. A. Garcés, J. C. Gómez-Izquierdo, and F. Gonzalez-Canales, Eur. Phys. J. C 78, 812 (2018).

[19] A sampling is W. Grimus and L. Lavoura, J. High Energy Phys. 08 (2005) 013; R. Jora, J. Schechter, and M. Naeem Shahid, Phys. Rev. D 80, 093007 (2009); 82, 079902(E) (2010); Z. z. Xing, D. Yang, and S. Zhou, Phys. Lett. B 690, 304 (2010); T. Teshima and Y. Okumura, Phys. Rev. D 84, 016003 (2011); S. Dev, S. Gupta, and R. R. Gautam, Phys. Lett. B 702, 28 (2011); S. Zhou, Phys. Lett. B 704, 291 (2011); R. Jora, J. Schechter, and M. N. Shahid, Int. J. Mod. Phys. A 28, 1350028 (2013); H. B. Benaoum, Phys. Rev. D 87, 073010 (2013).

[20] S. Morisi, arXiv:hep-ph/0605167; M. Tanimoto and T. Yanagida, Phys. Lett. B 633, 567 (2006); S. Gupta, C. S. Kim, and P. Sharma, Phys. Lett. B 740, 353 (2015).
[21] A. E. C. Hernandez, E. C. Mur, and R. Martinez, Phys. Rev. D 90, 073001 (2014); V. V. Vien and H. N. Long, Zh. Eksp. Teor. Fiz. 145, 991 (2014) [J. Exp. Theor. Phys. 118, 869 (2014)]; E. Ma and R. Srivastava, Phys. Lett. B 741, 217 (2015); D. Meloni, S. Morisi, and E. Peinado, J. Phys. G 38, 015003 (2011); A. E. Carcamo Hernandez, I. de Medeiros Varzielas, and E. Schumacher, Phys. Rev. D 93, 016003 (2016); A. E. Carcamo Hernandez, I. de Medeiros Varzielas, and N. A. Neill, Phys. Rev. D 94, 033011 (2016); R. N. Mohapatra, S. Nasri, and H. B. Yu, Phys. Lett. B 639, 318 (2006); J. C. Gómez-Izquierdo and M. Mondragón, Eur. Phys. J. C 79, 285 (2019).

[22] F. Feruglio and Y. Lin, Nucl. Phys. B800, 77 (2008).

[23] G. Bhattacharyya, P. Leser, and H. Pas, Phys. Rev. D 83, 011701 (2011).

[24] D. Das, U. K. Dey, and P. B. Pal, Phys. Lett. B 753, 315 (2016); Phys. Rev. D 96, 031701 (2017); D. Das and P. B. Pal, Phys. Rev. D 98, 115001 (2018); D. Cogollo and J. P. Silva, Phys. Rev. D 93, 095024 (2016); F. Gonzlez Canales, A. Mondragón, M. Mondragón, U. J. Saldaa Salazar, and L. Velasco-Sevilla, Phys. Rev. D 88, 096004 (2013); A. E. Cárcamo Hernández, S. Kovalenko, and I. Schmidt, J. High Energy Phys. 02 (2017) 125; A. E. Cárcamo Hernández, Eur. Phys. J. C 76, 503 (2016).

[25] Kang, O. Popov, R. Srivastava, J. W. F. Valle, and C. A. Vaquera-Araujo, arXiv:1902.05966; N. Rojas, R. Srivastava, and J. W. F. Valle, Phys. Lett. B 789, 132 (2019); M. A. Daz, N. Rojas, S. Urrutia-Quiroga, and J. W. F. Valle, J. High Energy Phys. 08 (2017) 017; A. Merle, M. Platscher, N. Rojas, J. W. F. Valle, and A. Vicente, J. High Energy Phys. 07 (2016) 013; M. Hirsch, R. A. Lineros, S. Morisi, J. Palacio, N. Rojas, and J. W. F. Valle, J. High Energy Phys. 10 (2013) 149; C. Bonilla, E. Ma, E. Peinado, and J. W. F. Valle, Phys. Lett. B 762, 214 (2016).

[26] Y. Cai, J. Herrero-Garca, M. A. Schmidt, A. Vicente, and R. R. Volkas, Front. Phys. 5, 63 (2017); C. Klein, M. Lindner, and S. Ohmer, J. High Energy Phys. 03 (2019) 018.

[27] S. Pramanick, arXiv:1903.04208.

[28] E. Ma, Phys. Rev. D 73, 077301 (2006).

[29] N. G. Deshpande, M. Gupta, and P. B. Pal, Phys. Rev. D 45, 953 (1992). 